\title{
Dental Bleaching: An Overview
}

\author{
Megha Gupta ${ }^{1}$, Jatinder Kalotra ${ }^{2}$, Mayank Mall ${ }^{3}$, Shivani Targotra ${ }^{4}$, Puneet Gupta ${ }^{5}$, Anukriti ${ }^{6}$
}

\begin{abstract}
Tooth discoloration can often be corrected totally or partially by bleaching. Bleaching is most opted esthetic procedure because it is conservative, non-invasive, and inexpensive. Different materials and techniques have been proposed to achieve instant whitening with no risk and relapse. The purpose of this literature review is to explain the determinant factors that influence satisfactory final results of the techniques and provide a general overview, to achieve a treatment decision based on evidence.
\end{abstract}

Keywords: Carbamide, Hydrogen peroxide, Sensitivity, Stains, Vital tooth

Asian Pac. J. Health Sci., (2020); DOI: 10.21276/apjhs.2020.7.2.14

\section{INTRODUCTION}

Bleaching expands the scope of esthetic dentistry. It is less invasive method to lighten discolored teeth. ${ }^{[1]}$ Bleaching techniques may be classified as to whether they involve vital or non-vital teeth and whether the procedure is performed in office or outside the office. ${ }^{[2]}$ It is known that bleaching is based on a complex oxidation reaction in which oxygen free radicals, due to their low molecular weight, infiltrate through the enamel and dentin substrates. The carbamide peroxide (CP) concentration of commercial products varies from $10 \%$ to $22 \% .^{[1]}$

A lot of cosmetic dentistry is desired related. When literature is reviewed regarding use of whitening agents, it demonstrates that researchers have also quoted some undesirable effects caused by them along with whitening. If the long-term consequences and altered tooth biomechanics resulting from any cosmetic procedure before carrying out are ignored, it could have a disastrous effect on the image and credibility of our profession. ${ }^{[3]}$ Bleaching is mainly dependent on material and technique used.

\section{Constituents of Bleaching Gels ${ }^{[4]}$}

- $\quad \mathrm{CP}$ hydrogen peroxide and sodium hydroxide

- Non-hydrogen peroxide containing materials, that is, sodium perborate thickening agent Carbopol or polyx

- Urea

- Vehicle - glycerin, dentifrice, and glycol surfactant

- Preservatives flavoring fluoride.

1. $\mathrm{CP}$ (urea peroxide)

$\mathrm{CP}\left(\mathrm{CH}_{6} \mathrm{~N}_{2} \mathrm{O}_{3}\right)$ in $10 \%$ concentration is used in most of the home bleaching kits. This breaks down to $3.35 \%$ solution of hydrogen peroxide and $6.65 \%$ solution of urea. Hydrogen peroxide liberated from CP is effective in bleaching teeth. ${ }^{[5]}$ About $10 \%$ CP gel is safe and efficient for home tooth bleaching. Various other concentrations such as $15 \%, 20 \%$, and $35 \%$ solution are available. ${ }^{[6]}$

\section{Side effects: Mild laxative effect ${ }^{[3]}$}

2. Hydrogen peroxide

Hydrogen peroxide breaks down into water and oxygen. It is the oxygen molecules that penetrate the tooth and liberate the pigment molecule causing tooth to whiten. About 30\%
'Senior Lecturer, Department of Oral and Maxillofacial Surgery, MM College of Dental Sciences, Mullana, Haryana, India

${ }^{2} \mathrm{MDS}$, Conservative Dentistry and Endodontics, Kathua, Jammu and Kashmir, India

${ }^{3}$ MDS, Orthodontics and Dentofacial Orthopaedics, Rudraprayag, Uttarakhand, India

${ }^{4}$ PG Student, Department of Paedodontics and Preventive Dentistry, Swami Devi Dayal Dental College, Barwala, Haryana, India

${ }^{5} \mathrm{MDS}$, Orthodontics and Dentofacial Orthopaedics, Yamuna Nagar, Haryana, India

${ }^{6}$ Dental Surgeon, Ambala City, Haryana, India

Corresponding Author: Dr. Jatinder Kalotra, MDS, Conservative Dentistry and Endodontics, Kathua - 184101, Jammu and Kashmir, India.Email: jatinderkalotra@gmail.com

How to cite this article: Gupta M, Kalotra J, Mall M, Targotra S, Gupta P, Anukriti. Dental Bleaching: An Overview. Asian Pac. J. Health Sci., 2020 7(2):58-62

Source of support: Nil

Conflict of interest: None

Received: 18/04/2020 Revised: 30/05/2020 Accepted: 02/06/2020

hydrogen peroxide adversely influences the tensile bond strength of the $4^{\text {th }}$ generation bonding agent. ${ }^{[7]}$

3. Non-hydrogen peroxide containing material

Sodium perborate is the active ingredient. They also contain hydroxylite, sodium chloride, oxygen, sodium fluoride, and other raw materials. During the manufacturing process, an oxygen complex is created while eliminating sodium perborate. A peroxide free gel is produced in its final state. The gel interacts with moist tooth structure and is activated. The oxygen complex interacts with the tooth structure and saturates and changes the amino acids and double bonds of oxygen, which are responsible for tooth discoloration. In the presence of acid, warm air, or water, sodium perborate decomposes to form sodium metaborate, hydrogen peroxide, and nascent oxygen. It is most commonly used in intracoronal bleaching procedures. ${ }^{[8]}$

4. Thickening agents

a. Carbopol (carboxypolymethylene): This is a polyacrylic acid polymer. Trolamine, which is a neutralizing agent, is often added to Carbopol to reduce the $\mathrm{pH}$ of the gels to $5-7 \cdot{ }^{[8]}$

02020 The Author(s). This is an open access article distributed under the terms of the Creative Commons Attribution License (http:// creativecommons.org/ licenses/by/4.0/), which permits unrestricted use, distribution, and reproduction in any medium, provided the original work is properly cited. 
b. Polyx: It is the thickener used in the Colgate platinum system. ${ }^{[8]}$

5. Urea

It occurs naturally in the body. It is produced in the salivary glands and is present in saliva and gingival crevicular fluid. It breaks down to ammonia and carbon dioxide either spontaneously or through bacterial metabolism.

Urea is used in the bleaching kits to stabilize the hydrogen peroxide, enhances anticariogenic effects, saliva stimulation, and wound healing properties..$^{[9]}$

6. Vehicle

a. Glycerin: CP is formulated with a glycerin base, which enhances the viscosity of preparation. This may dehydrate the tooth

b. Dentifrice: Used as a vehicle for Colgate platinum system

c. Glycol: This is anhydrous glycerin. ${ }^{[8]}$

7. Surfactant

It functions as a surface wetting agent, which allows the hydrogen peroxide to diffuse across the gel tooth boundary. ${ }^{[10]}$

8. Flavorings

These are used in the bleaching materials to add to the choice of bleaching agent and to improve patient acceptability of product (e.g., melon, banana, and mint). ${ }^{[8]}$

\section{Side effects of bleaching materials ${ }^{[8]}$}

Gingival irritation, ulceration, and soreness, white spots or banding within tooth, sore throat, unpleasant taste, burning palate, pain, sensitivity, and gastric irritation

Mclnnes (bleaching) solution: It is one of the most commonly used bleaching solutions which is prepared by mixing most of the components of bleaching and was first prepared by Mclnnes.

Old: Containing 30\% hydrogen peroxide - bleaches enamel. $36 \%$ hydrochloric acid - etches enamel.

$0.2 \%$ ether - removes surface debris.

5:5:1 ratio.

New: $30 \%$ hydrogen peroxide.

$20 \%$ sodium hydroxide.

$0.2 \%$ ether - removal of surface debris.

Disadvantage:

1. Loss of contour

2. Sensitivity

3. Irritation to gingiva. ${ }^{[11]}$

\section{Bleaching of Vital Teeth}

a. In office bleaching of vital teeth (chair side bleaching, power bleaching, laser bleaching, dentist administered/applied bleaching, and assisted bleaching [Miller, 1999]/dentist supervised bleaching) ${ }^{[8]}$

i. Power bleaching:[12-14]

This is a term given to accelerate, in office tooth whitening procedures using either a xenon plasma arc curing light or a laser. Plasma arc lamp was introduced in 1993.

\section{Materials}

$35 \%$ hydrogen peroxide liquid.

$35 \%$ CP.

Various concentrations or combination of the above materials.
Dual activated bleaching system. This material contains $35 \%$ hydrogen peroxide gel, which is both light and chemically activated.

\section{Mechanism of power bleaching}

The technique works by lightening the enamel to give the appearance of whiteness. One theory is that the large colored organic molecules responsible for the stains are reduced to smaller, less noticeable molecules by a process of oxidation. The hydrogen peroxide acts both as an oxygenator and an oxidant.

Another theory is that the peroxide penetrates into enamel and dentin and oxidizes tooth discolorations. Nascent oxygen penetrates into enamel followed by dentin.

\section{Procedure}

Patient is assessed clinically and radiographically. Proposed treatment plan is discussed. Pre-operative photographs of teeth are taken. Teeth are isolated with a protective mucous membrane seal and gingiva is protected. Teeth are ligated with floss to protect the material from creeping under the dam. Teeth are cleaned with pumice prophylaxis paste. Bleaching material is now applied to the teeth. Light is applied close to the teeth. Teeth are then washed, and the bleach is reapplied for a further $10 \mathrm{~min}$ and the process is repeated for $45 \mathrm{~min}$ to $1 \mathrm{~h}$. Teeth are polished with diamond polishing paste or aluminum oxide discs. Dam is then removed. Mouth is rinsed and shade of the teeth is now assessed. A postoperative photograph can be taken. Patient is called 3-6 weeks later and this process can be repeated in 6 weeks increment till the desired shade is achieved.

\section{Benefits of power bleaching gels}

Less tooth sensitivity, tooth rehydration.

Indications: Mild generalized staining, age yellowing discoloration mild-to-moderate tetracycline staining, mild fluorosis, and color changes related to pulpal trauma or necrosis.

- Advantage

Less procedure time, removal of yellow brown stains.

- Disadvantage

Expensive, unpredictable response, frequent appointments, tooth dehydration, tissue burns, for example, lips, cheeks, and gingiva.

ii. Waiting room bleach technique (assisted bleach technique)

About 35\% CP (which breaks down to 10\% hydrogen peroxide) is marketed as a power bleaching agent. The teeth are polished with prophylaxis paste. Cheek and lip retractors are placed. About $35 \%$ CP can also be heated gently by holding the syringe under hot running water for 2-3 min. Heat of the syringe accelerates the activity of the material before it is loaded into the mouth guard. The dentist applies 35\% CP into a custom-made bleaching tray, followed by patient moving into waiting room for $30 \mathrm{~min}$ with bleaching tray in the mouth and then bleach is suctioned off the teeth before rinsing.

Other techniques such as compressive bleaching technique dual-activated technique, power bleaching techniques using heat, and the laser bleaching technique are more effective but less commonly used due to cost and feasibility.

b. The home bleaching technique (nightguard vital bleaching) ${ }^{[15,16]}$ 


\section{Protocol 1: Before commencing bleaching treatment, the} following assessments are done

- Patients medical history, ascertain which foods, or drinks are causing the staining of the teeth

- Medication intake

- $\quad$ Smokers should be asked to stop smoking at least a week before, not smoke during the bleaching treatment, which could take up to 1 month, and not smoke for at least a month after.

\section{Initial consultation}

- The internal discussion with the patient should address

- Advantages and disadvantages of tooth bleaching alternatives to bleaching treatments

- Any side effects that may be experienced

- Risks and benefits of the procedures and obtainment of the patient informed consent

- Duration of treatment

- Further esthetic treatment and dental treatment that may be needed.

\section{Clinical examination of all teeth}

Assess the oral environment, soft tissues, mucosa, teeth, gingiva, and oral health status of the patient. Check the integrity of the existing restorations. Vitality of teeth should be tested. Teeth that are non-vital should be root treated with a good apical seal. Cervical recession, periodontal health, and any cracking of the anterior teeth should be assessed.

\section{Pre-existing shade evaluations}

Shade taking can be through the normal methods, that is, using the porcelain shade guide or through the shade guide supplied with the bleaching kit.

\section{Treatment planning}

Bleach only one arch at a time. Tell the patient that the existing composite restoration may not match after bleaching and that it may be necessary to replace these composites with lighter ones after the bleaching procedures. Photographs together with the existing shade tab of the teeth are taken.

\section{Impression taking}

Excellent impressions reproducing the surfaces of the upper and lower teeth should be taken so that the bleaching trays can be made. Alginate or another accurate material can be used.

\section{Seating the tray}

- $\quad$ For a full arch

The bleaching trays are checked for correct fit, retention, and over extension on the gingival area. The amount of bleaching material to be used can be demonstrated to the patient and the patient is helped to insert and remove the trays. Patients are requested to brush and floss their teeth and then to apply the tray with the bleaching material in their mouth. Excess is removed with cotton roll, finger, or toothbrush. They can choose to either bleach their teeth while they sleep or to apply the tray during the day.

Normally, it is best to start with the bleaching of the maxillary teeth first as these bleach quicker. This is thought to be due to better retention of the upper tray, effects of gravity, and reduced effects of salivary flow compared to the mandibular arch.

- For single teeth

Full arch trays are constructed but patients are instructed to only place the bleaching material in the location of the dark tooth. To help patients identify where to place the bleaching material, a small notch can be cut into tray above the tooth.

It is best to review the patient 1-2 weeks after wearing the trays. Monitor the oral environment, soft tissue, mucosa, gingival health, and teeth for any adverse reactions.

\section{Maintenance after tooth bleaching}

Once the light color has been achieved and patient is satisfied with the color, bleaching treatment is terminated. The trays are returned and kept at the dental office. Patient should continue their regular maintenance visits, which includes periodic oral health evaluation. The advantage of home bleaching is that should the teeth darken slightly, rebleaching is easy, providing the trays still fit correctly and do not distort. Additional rebleaching and/or top bleaching can be done every $3-4$ years if necessary. ${ }^{[15,16]}$

\section{Tetracycline stained teeth}

The intensity and pattern of discoloration are dependent on the dosage, duration, and type given, as well as the calcification activity of the teeth. Staining can be generalized or localized in horizontal bands within the tooth. Home bleaching treatment for tetracycline works more slowly than for age-yellowed teeth, but successful lightening and whitening can be achieved over an extended period from 3 to 6 months. ${ }^{[8]}$

\section{Side effects and problems}

Gingival irritation, soft-tissue irritation, altered taste sensation, and tooth thermal sensitivity.

- Advantages

Simple and fast to use, cost effective, painless procedure, patients convenient, quick results, and satisfaction

- Disadvantages

Active patient participation

Color change is dependent on the amount of time that the trays are worn. If patients do not wear the bleach in the trays for the specified amount of time, change in tooth lightening will be slow Excessive amounts of bleach for too many hours per day.

\section{Indications}

Mild generalized staining, age yellowing discoloration, mild tetracycline staining, very mild fluorosis, stains from smoking tobacco, color change due to pulpal trauma or necrosis, and young patients with an inherited gray or yellow hue to the teeth.

\section{Contraindications}

Severe tetracycline staining, pitting hypoplasia, fluorosis stain, and discolorations in the adolescent patient with large pulp teeth with inadequate or defective existing restorations 


\section{Teeth with}

Surface loss due to attrition, abrasion and erosion, with insufficient enamel that respond to bleaching, with deep and surface cracks and fracture lines, with large anterior restorations, Pathology such as periapical radiolucency.

Fractured or maligned teeth, pregnancy, lactation and smoking, teeth sensitivity to heat, cold, touch, and sweet. ${ }^{[15,16]}$

Fabrication of home bleaching trays: ${ }^{[17]}$

- Good alginate impression is taken

- Cast a model

- Trimming of model

- Block out resin is placed

- Resin curing

- Application of separator/cold mold seal

- Tray sheet material is selected

- Cast the plastic in the vacuum tray-forming machine

- Trimming of tray

- $\quad$ Polishing of tray.

\section{Bleaching of Non-vital Teeth}

Intracoronal bleaching of non-vital teeth involves the use of chemical agents within the coronal portion of endodontically treated teeth to remove tooth discoloration. The successful outcome depends mainly on the etiology, correct diagnosis, and proper selection of bleaching technique.

Methods most commonly employed to bleach endodontically treated teeth are the walking bleach and thermo/photobleaching techniques. ${ }^{[17]}$

\section{Walking bleach procedures: ${ }^{[17,18]}$}

Walking bleach is preferred since it requires less chair time and is safer and more comfortable to patients. Mixture of sodium perborate and superoxol, decomposes into sodium metaborate, water, and oxygen. When sealed in pulp chamber, oxidizes and discolors the stains slowly. This is walking bleach.

\section{Technique}

- Familiarize the patient with possible causes of discoloration, procedures to be followed, expected outcome, and possibility of future discoloration

- $\quad$ Radiographically assess the status of the periapical tissues and quality of endodontic obturation; endodontic failure or questionable obturation should always be retreated prior to bleaching

- Defective restorations should be replaced

- Evaluate tooth color with a shade guide and if possible, take clinical photographs at the beginning and throughout the procedure

- Isolate the tooth with a rubber dam. The dam must fit tightly at the cervical of the tooth to prevent possible leakage of bleaching agent into gingival tissue. Prior to dam placement protective cream, such as Orabase or Vaseline, must be applied to the surrounding gingival tissues

- Remove the restorative material from the access cavity, expose the dentin, and refine the access

- $\quad$ Remove all materials to a level just below the labial gingival margin. Orange solvent, chloroform, or xylene on a cotton pellet may be used to dissolve sealer remnants
- Apply a sufficient thick layer, at least $2 \mathrm{~mm}$ of a protective white cement barrier such as polycarboxylate cement, zinc phosphate cement, glass ionomer, Cavit, or IRM to cover the endodontic obturation

- Walking bleach paste is prepared by mixing sodium perborate and an inert liquid such as water, saline, or anesthetic solution, to a thick consistency of wet sand and packed in pulp chamber. Remove excess liquid by tamping with a cotton pellet. This also compresses and pushes the paste into all areas of pulp chamber

- $\quad$ Excess bleaching paste is removed from undercuts in pulp horn and gingival area and thick, well-sealed temporary filling is applied directly against the paste and into the undercuts. Carefully pack the temporary filling, at least $3 \mathrm{~mm}$ thick, to ensure a good seal

- $\quad$ Remove the rubber dam and inform the patient that bleaching agent works slowly

- $\quad$ Evaluate the patient 2 weeks later and if necessary repeat the procedure several times.

It should be noted that increasing the amount of bleaching increases the risk of cervical resorption and should be minimal provided that a barrier should be placed in CEJ where it may cause severe sensitivity. ${ }^{[19]}$

\section{Thermo catalytic/photobleaching procedure: ${ }^{[18,20]}$}

The technique involves placement of the oxidizing, chemical bleaching material generally $30-35 \%$ hydrogen peroxide in pulp chamber, followed by either heat application by electric heating devices, light application by specially designed lamps, or both.

\section{Technique}

- Familiarize the patient with the probable cause of discoloration, the procedures to be followed, expected outcome, and possibility of future discoloration

- Take radiographs to assess the status of periapical tissues and quality of endodontic obturation

- Tooth color is evaluated with a shade guide

- Apply protective cream to the surrounding gingival tissues and isolate the teeth with a rubber dam and waxed dental floss ligatures to the patient

- Do not use anesthesia

- Position protective sunglasses over patients and operators eyes

- Apply a sufficiently thick layer, at least $2 \mathrm{~mm}$ of protective white cement barrier, such as polycarboxylate cement, zinc phosphate cement, glass-ionomer, intermediate restorative material, or Cavit on the endodontic obturation

- Soak a small amount of 30-35\% hydrogen peroxide solution on a small cotton pellet or a piece of gauze and place it into the pulp chamber. A bleaching gel containing hydrogen peroxide may be used instead of aqueous solution

- Apply heat with a heating device or a light source. The temperature should be between 50 and $60^{\circ} \mathrm{C}$ so that patient can tolerate it comfortably

- $\quad$ Rewet the cotton pellet and pulp chamber with hydrogen peroxide as necessary. If tooth becomes too sensitive, discontinue bleaching procedure immediately

- Remove the heat or light source and allow the teeth to cool down for at least $5 \mathrm{~min}$. Then wash with warm water for $1 \mathrm{~min}$ and remove the rubber dam 
- $\quad$ Dry the tooth and place walking bleach paste into the pulp chamber

- Recall the patient approximately 2 weeks later and evaluate the effectiveness of bleaching. Take clinical photographs with the same shade guide used in the pre-operative photographs for comparison purposes.

\section{Conclusion}

The increasing demand for tooth bleaching has driven many manufacturers and researchers to develop bleaching products to be used either in the dental office or at home. However, as with any dental procedure, bleaching involves risks. It should be accompanied by other esthetic procedures for better results. Finally, clinicians should inform their patients about the possible changes that may occur on their dental restorations during bleaching procedure as well as the possibility of replacement of the bleached restorations at the end of bleaching treatment.

\section{References}

1. Kaya AD, Turkun M. Reversal of dentin bonding to bleached teeth. Oper Dent 2003;28:825-9.

2. Carrillo A, Veronica M. Simultaneous bleaching of vital teeth and an open chamber non vital tooth with $10 \%$ carbamide peroxide. Quintessence Int 1998;29:643-8.

3. Carey CM. Tooth whitening: What we now know. J Evid Based Dent Pract 2014;14 Suppl:70-6.

4. Alqahtani MQ. Tooth-bleaching procedures and their controversial effects: A literature review. Saud Dent J 2014:26:33-46.

5. Braun A, Jepsen SS, Krause F. Spectrophotometric and visual evaluation of vital tooth bleaching employing different carbamide peroxide concentrations. Dent Mat 2007;23:165-9.

6. Barcellos DC, Benetti P, Junior VV, Valera MC. Effect of carbamide peroxide bleaching gel concentration on the bond strength of dental substrates and resin composite. Oper Dent 2010;35:463-9.

7. Matis BA, Hamdan YS, Cochran MA, Eckert GJ. A clinical evalution of a bleaching agent used with and without reservoirs. Oper Dent 2002;27:5-11.

8. Greenwall L. Bleaching Techniques in Restorative Dentistry. $1^{\text {st }}$ ed. Boca Raton, Florida: CRC Press; 2001.

9. Moss SJ. Carbamide and food a food of the literature. FDI World 1999;3:9-14.

10. Feinman R, Madray G, Yarborough D. Chemical, optical and physiologic mechanisms of bleaching products: A review. Pract Periodont Aesthetic Dent 1991;3:32-7.

11. Jones $A H, A$ na M, Arnold D. Colorimetric assessment of laser and home bleaching techniques. J Esthet Dent 1999;11:87-94.

12. Sulieman M. An overview of bleaching techniques: 3 . In-surgery or power bleaching. Dent Update 2005;32:101-8.

13. Sulieman M, Addy M, MacDonald E, Rees JS. The bleaching depth of a 35\% hydrogen peroxide based in-office product: A study in-vitro. J Dent 2004;33:33-40.

14. Barghi NB. Making a clinical decision for vital tooth bleaching: At-home or in-office? Compend Contin Educ Dent 1998;19:831-8.

15. Sulieman M. An overview of bleaching techniques: 2. Night guard vital bleaching and non-vital bleaching. Dent Update 2005;32:39-46.

16. Haywood VB. Nightguard vital bleaching: Indications and limitations. US Dent 2006;1:1-8.

17. Goldstein's RE. Esthetics in Dentistry. $3^{\text {rd }}$ ed. Hoboken, USA: John Willey and Sons Inc.; 2018.

18. Plotino G, Buono L, Grande NM, Pameijer CH, Somma F. Non vital tooth bleaching: A review of the literature and clinical procedures. J Endod 2008;34:394-07.

19. Thakur K, Bhat N, Bharadwaj N, Bansal S. Microscopic analysis of the variations of cemento-enamel junction in Himachali population. Int J Health Clin Res 2019;2:1-4.

20. Baratieri LN, Ritter AV, Monteiro S Jr., Andrada MA, Vieira LC. Nonvital tooth bleaching: Guidelines for the clinician. Quintessence Int 1995;26:597-8. 